\title{
Grand unified parity solution to the strong $C P$ problem
}

\author{
Yukihiro Mimura, ${ }^{1}$ Rabindra N. Mohapatra, ${ }^{2}$ and Matt Severson ${ }^{2}$ \\ ${ }^{1}$ Institute of Science and Engineering, Shimane University, Matsue 690-8504, Japan \\ ${ }^{2}$ Maryland Center for Fundamental Physics, Department of Physics, University of Maryland, \\ College Park, Maryland 20742, USA
}

(Received 21 March 2019; published 19 June 2019)

\begin{abstract}
A beyond the standard model theory that respects parity symmetry at short distances is known to provide a solution to the strong $C P$ problem without the need for an axion, while keeping the Cabibbo-KobayashiMaskawa phase unconstrained. In this paper we present a supersymmetric $\operatorname{SO}(10)$ grand unified embedding of this idea with Yukawa couplings generated by 10, $\overline{\mathbf{1 2 6}}$ and $\mathbf{1 2 0}$ Higgs fields. This model is known to provide a unified description of masses and mixings of quarks and leptons. When $C P$ symmetry is imposed on this model, the discrete gauge subgroup $\mathrm{C}$ of $\mathrm{SO}(10)$ combines with it to generate an effective parity symmetry, leading to Hermitian quark mass matrices. Imposing an additional discrete symmetry, $G$, we show that there are no other tree level sources of $\theta$ in the model; $G$ also guarantees that the one- and two-loop contributions to $\theta$ vanish. We then show that the leading three-loop effects and the effect of higher-dimensional operators invariant under $G$ give rise to $\theta$ near the current experimental bound, making the model testable in the current searches for neutron electric dipole moment.
\end{abstract}

DOI: $10.1103 /$ PhysRevD.99.115025

\section{INTRODUCTION}

One of the major puzzles of the standard model is understanding the smallness of the QCD -induced $C P$ violating parameter $\theta$, which is bounded to be $\leq 10^{-10}$ from the electric dipole moment limits on the neutron. A popular solution to this puzzle is the Peccei-Quinn theory [1] which predicts the existence of a light pseudoscalar particle, the axion [2], with model dependent mass and couplings. The most widely discussed example is the so-called invisible axion suggested in [3]. There have been numerous attempts to experimentally discover the axion, and many new techniques have been proposed recently to search for it. So far such attempts have been unsuccessful.

Several alternative solutions to the $\theta$ problem that do not predict an axion have therefore been discussed in the literature [4]. A nontrivial problem here is to make $\theta$ so tiny while keeping the Cabibbo-Kobayashi-Maskawa (CKM) phase so large. One of the earliest classes of solutions without an axion [5,6] is based on the left-right symmetric theories (LRSM) [7], which extend the standard model to provide a framework for understanding the origin of parity violation. These theories conserve parity $(P)$ prior to symmetry breaking, which implies that the quark

Published by the American Physical Society under the terms of the Creative Commons Attribution 4.0 International license. Further distribution of this work must maintain attribution to the author(s) and the published article's title, journal citation, and DOI. Funded by SCOAP ${ }^{3}$.
Yukawa coupling matrices are Hermitian if parity transformation $P$ is defined as $Q_{L} \rightarrow Q_{R}$. If the vacuum expectation values (vevs) of the Higgs fields that break the standard model gauge symmetry are real, the resulting quark mass matrices will be Hermitian, leading to $\arg \operatorname{det} M_{u} M_{d} \equiv \theta=0$ at tree level while the CKM phase remains unconstrained.

However, since parity symmetry must be broken at a scale higher than $v_{w k}$ to explain observed weak interactions, the question arises as to whether $\theta$ reappears after parity breaking. The answer is that nonzero $\theta$ does appear from quantum loops involving the parity-breaking scale, but the resulting value is finite, calculable, and small. Typically extra symmetries such as supersymmetry are needed [8] to suppress $\theta$ to the desired level. For further discussion of such models with supersymmetry, see [9].

The simplest complete parity-based model which does not require any extra symmetries is the quark seesaw version of LRSM [10] where $\theta$ arises at the two-loop level and is safely below the current neutron electric dipole moment limits $[10,11]$. Parity-based solutions that use a $U(1)$-extended version of this model have also been discussed in the literature [12-14].

Another class of widely discussed models uses spontaneously broken $C P$ invariance [15], but it has been argued $[4,16]$ that getting large CKM phase is more of a challenge in these models. For further discussion of these models see $[16,17]$.

In this paper, we focus on parity-based models and discuss their embedding into supersymmetric $\mathrm{SO}(10)$ grand 
unified theories. To the best of our knowledge, there is no supersymmetric grand unified theory (SUSY GUT) embedding of this kind of solution. For a supersymmetric bottomup model with coupling unification but not full GUT embedding; see [18] and a nonsupersymmetric GUT embedding based on $S O(10) \times C P$ group, see [11]. Nontriviality of supersymmetric GUT embedding comes from the fact that there are many beyond the standard model colored fermions from Higgs supermultiplets as well as matter multiplets which could potentially contribute an arbitrary amount to $\theta$, spoiling the strong $C P$ solution. Such contributions could arise if, for instance, there are phases in their superpotential couplings or in vacuum expectation values. Use of discrete symmetries helps us to overcome these problems in the model discussed here. We then show that corrections to $\theta$ arising from quantum effects as well as from higher dimensional operators are acceptably small.

Our solution uses a class of recently discussed renormalizable SUSY SO(10) models with Yukawa-generating Higgs superfields belonging to $\mathbf{1 0}, \overline{\mathbf{1 2 6}} \oplus \mathbf{1 2 6}$ and $\mathbf{1 2 0}$ representations. The fact that models of this type have the potential to solve the strong $C P$ problem was noted in [19] where it was pointed out that if $C P$ symmetry is imposed on the model, the quark mass matrices in this model are Hermitian. This is because $\mathrm{SO}(10)$ contains a $\mathrm{C}$-gauge symmetry as its subgroup, so additionally imposing $C P$ makes the model P-symmetric. Again, in this model, the value of CKM phase is unconstrained.

In this paper we propose a model with a discrete symmetry that can lead to sufficiently small $\theta$ with the following features: (i) we provide the superpotential that does not allow any new light states in the theory, maintaining coupling unification as in the minimal suoersymmetric standard model (MSSM); (ii) We analyze loop effects on the value of $\theta$ and show that a nonzero $\theta$ arises in the form of a gluino mass phase only at the three-loop level, which contributes a highly suppressed $\theta$, making it a true solution to the strong $C P$ problem. Needless to say the model also provides a fit to all fermion masses including neutrinos and predicts observable proton decay as has been already been discussed in $[19,20]$.

\section{THE MODEL}

We work with the SUSY SO(10) theory where Yukawa couplings of SM fermions are generated by Higgs multiplets belonging to $\mathbf{1 0}, \overline{\mathbf{1 2 6}}$ and $\mathbf{1 2 0}$ representations (denoted by $H, \bar{\Delta}, \Sigma$ respectively). The fermions of the model belong to the $\mathbf{1 6}$ dimensional spinor representation (denoted by $\psi$ ). The most general Yukawa superpotential can be written as

$$
W_{Y}=h_{i j} \psi_{i} \psi_{j} H+f_{i j} \psi_{i} \psi_{j} \bar{\Delta}+g_{i j}^{\prime} \frac{Z_{\psi}}{\Lambda} \psi_{i} \psi_{j} \Sigma,
$$

where $h, f$ are symmetric matrices, $g^{\prime}$ is an antisymmetric matrix, and $Z_{\psi}$ is a spurion singlet field. We now require this theory to have an additional $C P$ symmetry under which $\psi \rightarrow \psi^{*}, \quad H, \quad \bar{\Delta} \rightarrow H^{*}, \quad \bar{\Delta}^{*}, \quad Z_{\psi} \rightarrow Z_{\psi}^{*} \quad$ and $\Sigma \rightarrow-\Sigma^{*}$. Requirement of $C P$ invariance then implies that $h, f$ are symmetric and real matrices and $g^{\prime}$ is an imaginary antisymmetric matrix, i.e., $g^{\prime}=i g^{\prime \prime}$ with $g^{\prime \prime}$ real. We then define $g \equiv g^{\prime \prime}\left\langle Z_{\psi}\right\rangle / \Lambda$.

The symmetry breaking pattern is as follows: at GUT scale, both $\mathrm{SO}(10)$ and parity symmetry break but SUSY is unbroken. SUSY breaking appears as soft scalar and guino masses at the $\mathrm{TeV}$ scale. As we show below the vevs of fields that break this symmetry are real. The MSSM doublets appear below the GUT scale as linear combination of the ones contained in $H, \bar{\Delta}, \Delta, \Sigma$ and the two up and down type MSSM doublets will be kept at the weak scale by the fine tuning of the Higgs superpotential parameters as is done in usual SUSY GUT theories. After substituting the vevs of the resulting MSSM doublets, we have (see Ref. [19] for convention)

$$
\begin{aligned}
\mathcal{M}_{u} & =\tilde{h}+r_{2} \tilde{f}+i r_{3} \tilde{g}, \\
\mathcal{M}_{d} & =\frac{r_{1}}{\tan \beta}(\tilde{h}+\tilde{f}+i \tilde{g}), \\
\mathcal{M}_{e} & =\frac{r_{1}}{\tan \beta}\left(\tilde{h}-3 \tilde{f}+i c_{e} \tilde{g}\right), \\
\mathcal{M}_{\nu_{D}} & =\tilde{h}-3 r_{2} \tilde{f}+i c_{\nu} \tilde{g}, \\
\mathcal{M}_{\nu} & =f v_{L}-\mathcal{M}_{\nu_{D}}\left(f v_{R}\right)^{-1} \mathcal{M}_{\nu_{D}}^{T},
\end{aligned}
$$

where $\tan \beta=v_{u} / v_{d}$ for vevs $v_{u, d}$ of the MSSM fields $H_{u, d}$. For $\lambda=h, f, g$, the couplings $\tilde{\lambda}_{i j}$ are related to $\lambda_{i j}$ by [19]

$\tilde{h} \equiv \mathcal{V}_{11} h v_{u} ; \quad \tilde{f} \equiv \frac{\mathcal{U}_{14} f v_{u}}{r_{1} \sqrt{3}} ; \quad \tilde{g} \equiv \frac{\mathcal{U}_{12}+\mathcal{U}_{13} / \sqrt{3}}{r_{1}} g v_{u}$.

where $\mathcal{V}, \mathcal{U}$ are the MSSM Higgs doublet mixing matrices at the GUT scale [19].

If we can guarantee that the mixings $\mathcal{U}, V_{i j}$ and vevs $v_{u, d}$ and $\left\langle Z_{\psi}\right\rangle$ are real, the quark and lepton mass matrices will be hermitian, i.e., $M_{q}=M_{q}^{\dagger}$, which implies that at tree level, $\theta=\arg \operatorname{det}\left(M_{u} M_{d}\right)=0$. At the same time, because of $i \tilde{g}$ term in the quark mass matrices, the CKM phase is arbitrary.

If this model is to be a solution to the strong $C P$ problem, we have to show the following:

(i) the mixing parameters $\mathcal{U}, V_{i j}$ in MSSM doublets which result from various GUT scales are real;

(ii) the masses of all the GUT-scale colored fermions are real (e.g., $\arg \operatorname{det} M_{C}=0$ for colored-Higgs mass matrices $M_{C}$ );

(iii) there are no higher order loop corrections to $\theta$ that are large; and

(iv) the full superpotential is such that there are no dangerous sub-GUT-scale multiplets that affect coupling unification. 
TABLE I. Charge assignment of the different superfields of the theory. It is understood that complex conjugate superfields have opposite discrete quantum numbers.

\begin{tabular}{lcc}
\hline \hline Field & $C P$ transformation & $\left(Z_{N_{1}} \times Z_{N_{2}} \times Z_{N_{3}}\right)$ charges \\
\hline$\Psi(16)$ & $\Psi^{*}(16)$ & $(-1 / 2,0,0)$ \\
$H(10)$ & $H^{*}(10)$ & $(1,0,0)$ \\
$\bar{\Delta}(\overline{126})$ & $\bar{\Delta}(\overline{126})^{*}$ & $(1,0,0)$ \\
$\Delta(126)$ & $\Delta(126)^{*}$ & $(1,1,0)$ \\
$\Sigma(120)$ & $-\Sigma(120)^{*}$ & $(1,0,1)$ \\
$A(45)$ & $-A(45)^{*}$ & $(-2,0,-1)$ \\
$S(54)$ & $S(54)^{*}$ & $(4,0,2)$ \\
$X_{\Delta}, \Phi(210)$ & $X_{\Delta}^{*}, \Phi(210)^{*}$ & $(-2,-1,0)$ \\
$X_{\Sigma}$ & $X_{\Sigma}^{*}$ & $(-8,0,-4)$ \\
$X_{S}$ & $X_{S}^{*}$ & $(4,0,2)$ \\
$X_{A}$ & $X_{A}^{*}$ & $(4,2,0)$ \\
$X_{\Phi}$ & $(0,0,-1)$ \\
$Z_{\psi}$ & $X_{\Phi}^{*}$ & $(-6,0,-2)$ \\
$Z_{H}$ & $Z_{\psi}^{*}$ & $(6,3,0)$ \\
$Z_{\Phi}$ & $Z_{H}^{*}$ & $(0,0,0)$ \\
$\lambda($ Gaugino $)$ & $Z_{\Phi}^{*}($ Gaugino $)$ & \\
\hline \hline
\end{tabular}

We show all these below. Clearly the first two require that all the couplings in the Higgs superpotential $\mathcal{W}$ are real. We show by an appropriate choice of the discrete symmetry $G$ and choice of $C P$ properties of the superfields that this condition is indeed satisfied in our model.

\section{SUPERPOTENTIAL}

In addition to the above multiplets which play a role in generating fermion masses, we add the following multiplets: 45, 54 and 210 (denoted by $A, S$ and $\Phi$ respectively). The $C P$ transformations of the various field in the model are given in the Table I. In column 3 of the Table, we give the transformation of the fields under a discrete group $G \equiv Z_{N_{1}} \times Z_{N_{2}} \times Z_{N_{3}}$. The purpose of the discrete group is to ensure that there are no complex parameters in the superpotential so that there will be at least one vacuum state which will have real vevs for the spurion fields forbidding any new contributions to the $\theta$ parameter. We have also used some of the Yukawa couplings and masses as spurion fields so that they have appropriate charges under $G$ to make that desired field term $G$-invariant. We will show that the spurions will acquire GUT scale vevs to generate the Yukawa couplings and masses of the right order and discuss how those vevs for the gauge singlet spurion fields arise.

In Table I, we have assigned the charges to the couplings and masses so that

(i) The Higgs doublets mixed by $H \Sigma A, \bar{\Delta} \Sigma A$ terms with a vev of $A$, as well as $S A A$, are allowed by the renormalizable coupling. (ii) The Yukawa coupling to $\Sigma$ can be suppressed so the charge of $\Sigma$ can be different from $H$ (we certainly want to write $\psi ж \psi H$ Yukawa coupling, which generates the top mass, as a renormalizable term).

(iii) The masses of $\Delta, A$, and $S$, as well as some couplings such as $\mathrm{SHH}$, are treated as spurion singlet fields.

The superpotential invariant under $S O(10) \times C P \times G$ [in addition to the Yukawa-like terms in Eq. (1)] is given by:

$$
\begin{aligned}
\mathcal{W}= & \sum_{\varphi} X_{\varphi} \varphi^{2}+X_{\Delta} \Delta \bar{\Delta}+\lambda_{2} \Sigma A H \\
& +\lambda_{4} \bar{\Delta} A \Sigma+\lambda_{5} S A A+\lambda_{6} H \Delta \Phi+Z_{H} S H H / \Lambda \\
& +\lambda_{7} \Phi \Delta \bar{\Delta}+\alpha_{0} Z_{\Phi} \Phi^{3} / \Lambda,
\end{aligned}
$$

with $\varphi=\Sigma, S, A, \Phi$. Note that in this superpotential, all the coupling parameters are real due to $C P$ invariance and the scale $\Lambda$, which is assumed to be the string scale, is much larger than the unification scale $M_{U}$. The reality of the couplings implies that all the GUT scale vevs of Higgs fields and the spurions will be real, as will be the mixing parameters $\mathcal{U}, V_{i j}$. Moreover, all the colored Higgs fields will have real mass matrices so that they will not contribute any new phase to the tree level $\theta$ parameter. This establishes our condition (i) and (ii) above at the leading order in the superpotential. Higher order nonrenormalizable terms can still disturb the conditions (i) and (ii); we will show that their contributions to theta are at or below the current bound.

Additionally, we choose the specific symmetry $G=$ $Z_{24} \times Z_{6} \times Z_{4}$ so that we can add the following superpotential terms:

$$
\begin{aligned}
W^{\prime}= & \alpha_{1} \frac{\left(X_{\Delta} \Delta \bar{\Delta}\right)^{2}}{\Lambda^{3}}+\alpha_{2} \frac{S^{6}}{\Lambda^{3}}+\alpha_{3} X_{S}^{3}+\alpha_{4} \frac{Z_{\Phi}^{4}}{\Lambda}+\alpha_{5} \frac{Z_{\Phi} X_{\Delta}^{3}}{\Lambda} \\
& +\alpha_{6} \frac{X_{A}^{6}}{\Lambda^{3}}+\alpha_{7} \frac{Z_{\psi}^{4}}{\Lambda}+\alpha_{8} \frac{Z_{\psi}^{2} X_{A} X_{\Sigma}^{2}}{\Lambda^{2}}+\alpha_{9} \frac{Z_{H}^{3} X_{\Sigma}^{3}}{\Lambda^{3}}+\alpha_{10} \frac{Z_{H}^{4}}{\Lambda} \\
& +\alpha_{11} \frac{X_{\Sigma}^{6} Z_{H}^{2}}{\Lambda^{5}}+\alpha_{12} \frac{X_{S}^{2} Z_{H} X_{\Sigma}}{\Lambda}+\alpha_{13} X_{A}^{2} X_{S}+\alpha_{14} \frac{X_{\Phi}^{3} Z_{\Phi}^{2}}{\Lambda^{2}} \\
& +\alpha_{15} X_{\Phi} X_{\Delta}^{2}+\alpha_{16} \frac{X_{\Phi}^{6}}{\Lambda_{3}}+(\text { higher order terms in } 1 / \Lambda) .
\end{aligned}
$$

With very mild fine tuning of the $\alpha_{i}$ couplings, the F-term minimization can give real GUT scale vevs to all spurion fields as desired.

\section{GUT SYMMETRY BREAKING AND PARTICLE SPECTRA}

In this section we show by analyzing the superpotential that there are no undesirable light particles below the GUT 
scale that could destroy coupling unification. First note that the higher dimensional terms in Eq. (5), as well other terms, help to give vevs to all the spurion fields. If we choose the cutoff scale $\Lambda$ to be the reduced Planck scale $\left(2 \times 10^{18} \mathrm{GeV}\right)$, by appropriate choice of the coefficients of the higher dimensional terms, we can keep the spurion vevs near the GUT scale $\left(M_{U} \sim 2 \times 10^{16} \mathrm{GeV}\right)$. This will lead to spurion masses below the GUT scale, but being gauge neutral, they do not affect the running of gauge couplings. We have checked that with a mild smallness of the coefficients of the higher dimensional operators, we can keep all the singlet vevs near the GUT scale.

Next, due to the absence of $A \Delta \bar{\Delta}$ term, the F-flatness conditions of $\Delta, \bar{\Delta}, \Phi$ and $S, A$ are separated. The $\Delta$ vev, which is at GUT scale along the $\mathrm{SU}(5)$-singlet direction i.e., $\left\langle\Delta_{13579}\right\rangle=\left\langle\bar{\Delta}_{13579}\right\rangle=v_{R} \neq 0$ (to get the D-terms to be zero), can be generated by $\left(\Phi+X_{\Delta}\right) \Delta \bar{\Delta}+\Phi^{3}+X_{\Phi} \Phi^{2}$ term. The vevs of $A$ can be generated by $X_{A} A^{2}+X_{S} S^{2}+$ $S A A$. For group theory of such models see [21,22].

Note that in the absence of the $\Phi \Delta \bar{\Delta}$ term, the superpotential is only a function of the singlet contraction of $\Delta \bar{\Delta}$, which implies that the superpotential has a large global symmetry, and thus the decomposed multiplets under $\mathrm{SU}(5)$ are massless. However, the presence of $\Phi \Delta \bar{\Delta}$ term cures this problem and makes all submultiplets of $\Delta \oplus \bar{\Delta}$ massive.

\section{A. Proton decay from higher dimensional operators}

In view of the fact that we have chosen $\Lambda \sim 2 \times 10^{18} \mathrm{GeV}$, one might wonder whether the model leads to rapid proton decay via higher dimensional operators scaled by $\Lambda$ as in usual SUSY GUT models. However, our model has a discrete symmetry $Z_{24} \times Z_{6} \times Z_{4}$, which all higher dimensional terms must also respect. As a result, the leading dangerous terms (for proton decay) such as $[\psi]^{4}$ are forbidden. The leading order term that contributes to proton decay after symmetry breaking is $\lambda[\psi \psi \bar{\Delta}]^{2}$ and this is suppressed by $\Lambda^{3}$ and as a result it quite is compatible with current limits on proton lifetime for $\lambda \leq 1$.

\section{SOLVING STRONG $\boldsymbol{C P}$}

We study the possible generation of the strong $C P$ phase, by (i) higher order terms, (ii) loop correction to the gluino mass, (iii) renormalization contribution to the quark Yukawa coupling as we extrapolate from GUT scale to the weak scale.

\section{A. Higher order terms}

In general one could envision two types of nonrenormalizable contribution to $\theta$ : (i) one set which are invariant under the discrete symmetry $C P \otimes G$ and (ii) terms induced by global symmetry-breaking, nonperturbative gravitational effects. We ignore the latter since there seem to be different opinions on whether black holes really break global symmetries.

Given the charge assignments in Table I, we find that the leading operators which can generate phases in the doublet Higgs mixings and the masses of colored Higgsinos are of dimension-9:

$$
\begin{aligned}
& A \Delta \bar{\Delta} X_{\Delta} Z_{H} Z_{\psi} S^{2} / \Lambda^{5}, \\
& H \Sigma \Phi \Delta \bar{\Delta} Z_{H} Z_{\psi} X_{A} / \Lambda^{5}, \\
& A \Phi \Phi X_{\Phi} Z_{H} Z_{\psi} X_{A}^{2} / \Lambda^{5},
\end{aligned}
$$

and the suppression factor will be $\left(M_{U} / \Lambda\right)^{5}$, where $M_{U}$ is a GUT unification scale $\sim 2 \times 10^{16} \mathrm{GeV}$. For $\Lambda$ to be the reduced Planck mass, $2.4 \times 10^{18} \mathrm{GeV}$, the bound for $|\theta|<$ $10^{-10}$ can be satisfied by these contributions to $\theta$.

It is also the case that a $\psi \psi A H$ term can break the Hermitian nature of the Yukawa coupling matrices, but such a term is generated only by a dimension- 10 operator under the above charge assignment, which leads to a $\theta$ below the current bound.

Note that the hierarchy between the cutoff (string scale) and the GUT unification is essential to suppress the $\theta$ parameter in the current model.

\section{B. Loop corrections and gluino phase}

Since phases in all colored fermion fields contribute to the $\theta$ parameter, we have to track the phases in the gluino mass term in addition to the quark and GUT-scale colored Higgsino mass matrices. At the tree level, gluino mass term is real due to $C P$ symmetry. However, since $C P$ symmetry is broken, quantum loops induce nonzero gluino phases. At the one-loop level, the gluino mass is real due to Hermiticity of the quark mass matrices, since the contribution from quarks is of the form $\operatorname{tr} M_{q}^{\dagger} A_{q}$ where $A_{q} \propto M_{q}$ within our setup and all other colored fermion fields have no phases.

The loop correction from the pure-imaginary Yukawa coupling $g$ can induce a phase for the gluino mass. At the two-loop level, however the contribution is always proportional to $\operatorname{Tr} Y Y^{\dagger}$, where $Y$ is a Yukawa coupling to colored Higgs, and the contribution is real. From the three-loop level diagram in which the doublet Higgs also propagates (Fig. 1), the imaginary part of the gluino mass is given by

$$
\sum_{a, b} \frac{g_{s}^{2}}{\left(16 \pi^{2}\right)^{3}} \operatorname{Tr}\left(Y_{T_{a}} Y_{D_{b}}^{*} Y_{\bar{T}_{a}} Y_{\bar{D}_{b}}^{*}\right) F\left(\frac{M_{H_{D_{b}}}}{M_{H_{T_{b}}}}\right) A_{\mathrm{tri}},
$$

where $Y_{T}$ and $Y_{\bar{T}}$ are Yukawa couplings to (diagonalized) colored Higgs fields $\left(H_{T, \bar{T}}\right)$, and $Y_{D}$ and $Y_{\bar{D}}$ are the Yukawa couplings to doublet Higgs fields $\left(H_{D, \bar{D}}\right)$, and $A_{\text {tri }}$ is the SUSY breaking scalar trilinear coupling, and $F$ is a loop function. (We note that the heavy Higgs doublets and the Higgs triplets with GUT scale mass propagate in the loop 


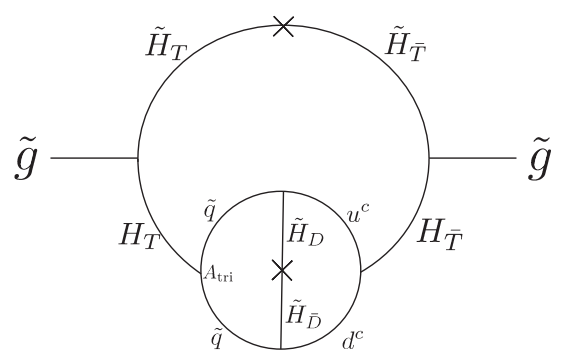

FIG. 1. The 3-loop diagram which induces the phase of the gluino mass. There are also diagrams in which $u^{c} e^{c}$-q $\ell$ and $\nu^{c} d^{c}-\ell q$ propagate. In $\mathbf{1 2 6}$ and $\mathbf{1 2 0}$, there are other colored components, e.g., (8, 2, 1/2), which have bifermion couplings, and there are similar loop-diagrams in which the other colored components propagate for the gluino mass correction.

diagram.) The Yukawa couplings are given by the linear combination of $h, f$ and $g$. Noting that $\operatorname{Tr}(g X)=0$ for symmetric matrix $X$, one finds that $\operatorname{Tr}\left(g h^{3}\right), \operatorname{Tr}(g h f h)$, etc vanish, and $\operatorname{Tr}(g h h f)$ and $\operatorname{Tr}(g f f h)$ etc. can contribute. As a result, the leading contribution can be estimated to be

$$
\operatorname{Im} m_{\tilde{g}} \sim \frac{g_{s}^{2} g_{23} f_{23} h_{33}^{2}}{\left(16 \pi^{2}\right)^{3}} A_{\text {tri }}
$$

For $A_{\text {tri }} \sim m_{\tilde{g}}$, we roughly estimate the contribution to $\theta$ as

$$
\Delta \theta \sim 10^{-9}\left|\frac{f_{23} g_{23}}{10^{-3}}\right| .
$$

This is on the borderline of satisfying the neutron electric dipole moment (EDM) bounds, taking into account that $f$ and $g$ are the original couplings and not multiplied by the Higgs mixing. Note that $\tilde{f}_{23}$ and $\tilde{g}_{23}$ (which are $f$ and $g$ multiplied by Higgs mixings) can be estimated to be as large as $V_{c b}$. If $A_{\text {tri }} \ll m_{\tilde{g}}$, the neutron EDM bounds can be safely satisfied; in this sense, gauge-mediated SUSY breaking, rather than the gravity mediation, is preferable to suppress the $\theta$ parameter. In any case, loop correction to the gluino mass can generate a borderline value for the $\theta$ parameter, and therefore, the model would predict an observable neutron EDM in current experiments. As for the squarks, their mass matrices are chosen diagonal at the GUT scale and while renormalization group extrapolations will induce some offdiagonal phases, they will not contribute to $\theta$ and any contribution to neutron EDM will be suppressed.

\section{Extrapolation of $\theta$ from GUT to weak scale}

The Hermiticity of the quark mass matrices holds at the GUT scale. This means that the value of tree level $\theta$ is zero at that scale and a finite, nonzero $\theta$ will be induced at the weak scale due to renormalization extrapolation of the various Yukawa. This issue for a parity solution to strong $C P$ has been considered in [18], and it is shown that if the weak scale theory is MSSM with a soft breaking scalar masses $M_{\text {SUSY }}$ above $5 \mathrm{TeV}$, the corrections to $\theta$ are given by

$\delta \theta \simeq\left(\frac{1}{16 \pi^{2}} \ln \frac{M_{U}}{M_{\mathrm{SUSY}}}\right)^{4}\left[c_{1} \operatorname{Im} \operatorname{Tr}\left[Y_{u}^{2} Y_{d}^{4} Y_{u}^{4} Y_{d}^{2}\right]+c_{2}(u \leftrightarrow d)\right]$.

This can be estimated to be $\delta \theta \sim 10^{-26}(\tan \beta)^{6}\left(c_{1}-c_{2}\right)$, which is below the experimental upper limit even for $\tan \beta=50$. We note that one obtains $c_{1}=c_{2}$ and $\delta \theta$ vanishes without an electroweak gauge loop at the MSSM scale and any extrapolation from there to the weak scale produces negligible change.

Finally, we note that since the discrete symmetries of our model break at the GUT scale, domain walls associated with them will get "inflated away" as long as the reheat temperature is below the GUT scale and will not lead to any anisotropy in cosmic microwave background.

\section{CONCLUSION}

In summary, we have proposed, for the first time, a consistent $\mathrm{SO}(10)$ grand unified embedding of the parity solution to strong $C P$ problem without the need for an axion. The model embeds the seesaw mechanism for neutrino masses and gives a fit to the fermion masses. The model predicts a neutron electric dipole moment not far from the current upper limits and can be testable in the near future.

\section{ACKNOWLEDGMENTS}

We thank Anson Hook for helpful discussions and comments on the manuscript. The work of Y. M. is supported by Scientific Grants by the Ministry of Education, Culture, Sports, Science and Technology of Japan (No. 16H00871, No. 16H02189, No. 17K05415 and No. 18H04590) and that of R.N.M. is supported by the U.S. National Science Foundation under Grant No. PHY1620074.
[1] R. D. Peccei and H. R. Quinn, Phys. Rev. Lett. 38, 1440 (1977).

[2] F. Wilczek, Phys. Rev. Lett. 40, 279 (1978); S. Weinberg, Phys. Rev. Lett. 40, 223 (1978).
[3] J. E. Kim, Phys. Rev. Lett. 43, 103 (1979); M. A. Shifman, A. I. Vainshtein, and V. I. Zakharov, Nucl. Phys. B166, 493 (1980); A. P. Zhitnitsky, Yad. Fiz. 31, 497 (1980) [Sov. J. Nucl. Phys. 31, 260 (1980)]; M. Dine, W. Fischler, and 
M. Srednicki, Phys. Lett. 104B, 199 (1981); For reviews, see J. E. Kim, Phys. Rep. 150, 1 (1987); J. E. Kim, S. Nam, and Y. K. Semertzidis, Int. J. Mod. Phys. A 33, 1830002 (2018).

[4] For a recent overview, see A. Hook, arXiv:1812.02669.

[5] M. A. B. Beg and H.-S. Tsao, Phys. Rev. Lett. 41, 278 (1978).

[6] R. N. Mohapatra and G. Senjanovic, Phys. Lett. 79B, 283 (1978).

[7] J. C. Pati and A. Salam, Phys. Rev. D 10, 275 (1974); R. N. Mohapatra and J. C. Pati, Phys. Rev. D 11, 566 (1975); 11, 2558 (1975); G. Senjanović and R. N. Mohapatra, Phys. Rev. D 12, 1502 (1975).

[8] R. Kuchimanchi, Phys. Rev. Lett. 76, 3486 (1996); R. N. Mohapatra and A. Rasin, Phys. Rev. Lett. 76, 3490 (1996); R. N. Mohapatra, A. Rasin, and G. Senjanovic, Phys. Rev. Lett. 79, 4744 (1997).

[9] A. Albaid, M. Dine, and P. Draper, J. High Energy Phys. 12 (2015) 046.

[10] K. S. Babu and R. N. Mohapatra, Phys. Rev. D 41, 1286 (1990).
[11] L. J. Hall and K. Harigaya, J. High Energy Phys. 10 (2018) 130.

[12] S. M. Barr, D. Chang, and G. Senjanovic, Phys. Rev. Lett. 67, 2765 (1991).

[13] A. Hook, Phys. Rev. Lett. 114, 141801 (2015).

[14] D. Dunsky, L. J. Hall, and K. Harigaya, arXiv:1902.07726.

[15] A. E. Nelson, Phys. Lett. 136B, 387 (1984); S. M. Barr, Phys. Rev. Lett. 53, 329 (1984).

[16] M. Dine and P. Draper, J. High Energy Phys. 08 (2015) 132.

[17] L. Vecchi, J. High Energy Phys. 04 (2017) 149.

[18] K. S. Babu, B. Dutta, and R. N. Mohapatra, Phys. Rev. D 65, 016005 (2001).

[19] B. Dutta, Y. Mimura, and R. N. Mohapatra, Phys. Rev. D 72, 075009 (2005).

[20] R. N. Mohapatra and M. Severson, J. High Energy Phys. 09 (2018) 119.

[21] T. Fukuyama, A. Ilakovac, T. Kikuchi, S. Meljanac, and N. Okada, J. Math. Phys. (N.Y.) 46, 033505 (2005); B. Bajc, A. Melfo, G. Senjanovic, and F. Vissani, Phys. Rev. D 70, 035007 (2004).

[22] C. S. Aulakh and S. K. Garg, Nucl. Phys. B857, 101 (2012). 